\title{
TRAYECTORIAS ESCOLARES Y TRAYECTORIAS LABORALES HACIA UNA ARTICULACION VIRTUOSA
}

\author{
María Valeria Álvarez* \\ Universidad Nacional de la Patagonia Austral \\ ma_rez@hotmail.com \\ María Lourdes Villanueva ** \\ Universidad Nacional de la Patagonia Austral \\ ma.lourdes.villanueva@gmail.com
}

Recibido: 16/06/2019 Aceptado: 11/12/2019

\section{Resumen}

Proponemos repensar la validez del mandato fundacional de la escuela, poniendo el acento en la formación para el trabajo. En un contexto de revisión de la escuela pública, sugerimos que formar trabajadores o articular trayectorias escolares con trayectorias laborales, pareciera ser una pretensión aún valiosa, con significativos impactos en las trayectorias biográficas de los sujetos aprendientes, y de la sociedad en general.

Al respecto, presentaremos argumentaciones y construiremos otras razones que fundamentan la vigencia de este mandato en nuestros días -más o menos convergentes-, intentando dar cuenta desde un marco fundamentalmente sociológico de los nuevos escenarios socio-económicos donde la escuela se sitúa, y los desafíos que las nuevas dinámicas implican para la educación, los individuos y el trabajo.

Reconocemos que es una articulación difícil y problemática donde los avances tecnológicos, y cambios en formas productivas, suponen en sí mismos desafíos para la política educativa, en tanto sus tiempos de ejecución no son exactamente coincidentes con las lecturas que pueda hacerse al respecto desde los diseños de políticas. Sin embargo, continúa siendo una respuesta o interpelación valiosa a la escuela, que creemos aún hoy, es una institución socialmente enriquecedora, y particularmente importante para los sujetos que la construyen y la habitan.

Palabras clave: Mandato escolar - Formación para el trabajo - Demandas del mercado laboral Política educativa - Nuevas tecnologías

\section{Abstract}

We propose to rethink the validity of the founding mandate of the school, emphasizing training for work. In a context of public school review, we suggest that training workers or articulating career paths with career paths seems to be a still valuable claim, with significant impacts on the biographical trajectories of learning subjects, and society in general.

*Profesora en Historia - Estudiante avanzada de la Maestría en Ciencias Sociales, orientación Sociología (Univ. De Quilmes) - Docente Adjunta - Área Historia Americana - Universidad Nacional de la Patagonia Austral, Unidad Académica Río Gallegos.

**Doctora en la orientación en Ciencias Sociales y Humanas (UNLU) - Licenciada en Sociología. Profesora Adjunta Área Metodología de la Investigación en Ciencias Sociales, orientación Educación, Universidad Nacional de la Patagonia Austral, Unidad Académica Río Gallegos. 


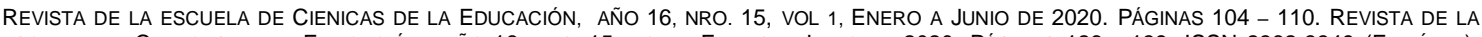
ESCUELA DE CIENICAS DE LA EDUCACIÓN, AÑO 16, NRO. 15, VOL 1, ENERO A JUNIO DE 2020. PÁGINAS 126 - 139. ISSN 2362-3349 (EN LíNEA). TRAYECTORIAS ESCOLARES Y TRAYECTORIAS LABORALES HACIA UNA ARTICULACIÓN VIRTUOSA. MARÍA VALERIA ÁlVAREZ. MARÍA LOURDES VILLANUEVA.

In this regard, we will present arguments and build other reasons that support the validity of this mandate in our days - more or less convergent -, trying to account from a fundamentally sociological framework of the new socio-economic scenarios where the school is located, and the challenges that the new dynamics imply for education, individuals and work.

We recognize that it is a difficult and problematic articulation where technological advances, and changes in productive forms, pose in themselves challenges for educational policy, while their execution times are not exactly coincident with the readings that can be made about it from the designs of policies. However, it remains a valuable response or interpellation to the school, which we still believe today, is a socially enriching institution, and particularly important for the subjects who build and inhabit it.

Keywords: School Mandate - Job Training - Labor Market Demands - Education Policy - New Technologies.

\section{Los individuos de la modernidad}

En la modernidad, el volverse individuo, -individualizarse- el resolver un proyecto de vida para sí mismo deviene una tarea siempre abierta y desafiante. En la modernidad clásica la integración social estaba dada por el trabajo remunerado generalmente masculino. A través del pleno empleo se garantizaba el ingreso a la ciudadanía social. Las políticas públicas universales (educación, sanidad, previsionales, entre otros) permitían un proyecto de vida con amplia previsibilidad (Castel, 2006).

En este sentido, los marcos colectivos de la segunda modernidad o modernidad tardía iniciada en los años 70' (clase, género y familia particularmente, de acuerdo a Beck, 2003), se tornan inseguros, siendo difícil, o al menos diluyéndose, donde anclarse para dar forma a la experiencia biográfica.

Así, indica Elias (1990) que en el camino de individualizarse, se toman decisiones importantes, sin tener la plena certeza de los resultados finales que dan forma a nuestra trayectoria vital. Al respecto indica Beck (2003) que la libertad de estos tiempos es precaria. Los marcos de referencia se diluyen: los movimientos feministas deconstruyen el género, la precarización laboral propia del neoliberalismo amenaza la clase, y las formas familiares se multiplican, siendo particularmente el matrimonio un espacio de negociación entre lo sujetos, que difícilmente perdure todas sus vidas. Por tanto, construirse a sí mismo es una tarea fatigosa -que exige un gran esfuerzo- y con escasas coordenadas fijas que orienten el camino, donde deben tomarse muchísimas decisiones.

Las oportunidades, los peligros y las incertidumbres biográficas que antes estaban predefinidas dentro de la asociación familiar o de la comunidad rural, o a tenor de las normativas de los estados o clases asistenciales, deben ahora percibirse, interpretarse, decidirse y procesarse por los propios individuos. Las consecuencias, tanto las oportunidades como las cargas, pasan ahora a los individuos que naturalmente, frente a la complejidad de las interrelaciones sociales, se ven a menudo incapaces de tomar las necesarias decisiones con el debido fundamento, ponderando los intereses, la moral y las posibles consecuencias. (Beck y Beck-Gernsheimp, 2003, p. 42)

Al respecto, una de nuestras hipótesis, es el reconocimiento de la escuela como institución socializadora clave para pensar el bagaje de herramientas laborales para enfrentar el proyecto personal. Sin embargo, y como adecuadamente indica Elias, el riesgo o la frustración que luego vive al adulto se origina muchas veces en el desajuste entre los anhelos socialmente formados por los jóvenes y las posibilidades de realizarlos.

Con la creciente especialización de las sociedades, al acrecentarse la distancia entre la espontaneidad y las actitudes esperadas en los adultos, ser más especializado el trabajo, más largo e indirecto es el proceso de preparación requerido a los jóvenes para insertarse en la sociedad, y también para habilitarlos a decidir por sí mismos. (Elias, 1990, p. 147)

En esta dirección, la formación para el trabajo es siempre beneficiosa para ingresar al mundo adulto. El trabajo podría ser así anclaje del sujeto, donde puede desplegar relativamente potencialidades y también obtener dinero para adquirir bienes y servicios en el mercado, reproducirse por tanto a sí mismo y a su familia. La formación transmitida debería ajustarse a las demandas reales o potenciales, para permitir una inserción efectiva y de relativa calidad.

\section{Las inseguridades del mercado laboral}

Si bien ha sido una pretensión de la escuela pública desde sus orígenes, la formación para el trabajo ha resultado siempre problemática y cambiante. Al respeto, los cambios en el mundo productivo y en su estructura tecnológica, por su naturaleza temporal, se anteponen y adelantan a las lecturas posibles que puede realizar la política educativa.

Desde fines de los años '70 asistimos a una nueva fase de las formas de producción industriales. Se han instalado dos nuevos paradigmas en las formas organizacionales: la integración para aumentar la productividad -reduciendo los tiempos muertos de las máquinas y de los consumos intermedios-, y la 
ReVista de la escuela de Cienicas de la Educación, AÑo 16, NRO. 15, vol 1, ENero a Junio de 2020. PÁGINAS 104 - 110. REVISTA DE LA ESCUELA DE CIENICAS DE LA EDUCACIÓN, AÑO 16, NRO. 15, VOL 1, ENERO A JUNIO DE 2020. PÁGINAS 126 - 139. ISSN 2362-3349 (EN LíNEA). TRAYECTORIAS ESCOLARES Y TRAYECTORIAS LABORALES HACIA UNA ARTICULACIÓN VIRTUOSA. MARÍA VALERIA ÁLVAREZ. MARÍA LOURDES VILLANUEVA.

flexibilidad de las líneas productivas. Ambas combinadas, responden a las presiones de los mercados y a las exigencias relativas a las mercancías, acorde a la nueva etapa de acumulación de capital (Coriat, 1993).

En este proceso convergen de modo clave los avances tecnológicos. El nacimiento de la microcomputadora en 1971, y posteriormente su penetración en el mundo de la técnica, impacta en el mundo productivo, reformando o supeditando otras tecnologías disponibles, siendo posteriormente más bien escasas aquellas que no suponen instancias de programación, uso de software, automatización y/o robótica. De este modo, la revolución de las tecnologías de la información (Coriat, 1993, p. 28; Stonier, 1979; en Morgan, 2000, p. 73), se articula con las tecnologías existentes, siendo por tanto entendida por Stonier como una "metatecnología".

Esta revolución es acompañada o sucede en el marco de un crecimiento de la desigualdad social a nivel mundial. La retracción del Estado en el marco del neoliberalismo, junto a la mercantilización de servicios y empresas públicas impactan en la configuración de un nuevo campo económico mundial. En consecuencia, de acuerdo con Foucault (1991, en Rodríguez, 2015), conviven zonas económicas diferenciadas: aquellas de alta rentabilidad e importante producción, donde prevalece la economía formal, y otras zonas que son mucho menos rentables y poco productivas donde se debilita la economía formal.

La intensificación de la competencia introduce tres formas de flexibilización de las empresas. La flexibilización técnica (Coriat, 1993, p. 52), asociada a la automatización programable, que permite variaciones en los productos y su volumen de acuerdo a la cantidad de demanda y gustos diferenciados de sus consumidores. Asimismo, se extiende la flexibilización externa y la flexibilización interna (Castel, 2006, p. 406). La primera refiere a las formas de subcontratación y tercerización aquellas fases de la producción que resultan más volátiles de acuerdo a la demanda del mercado. Mientras la segunda supone la polivalencia de la fuerza de trabajo. Surgen pequeños grupos de trabajadores que saben programar, controlan calidad y pueden reparar. Al respecto, diversos autores (Coriat, 1993; Castel, 2006) indican que son amplios los sectores sociales que portan saberes o formación que resultan desactualizados para la nueva demanda laboral; mientras existen segmentos que acceden a trabajos temporarios.

En este marco de mundialización de la economía crece a nivel mundial el sector servicios. Los mismos no son homogéneos en sus características de formalidad, y tipos de servicios ofrecidos. Muchas veces se presentan como opciones laborales, ante una reducción relativa de los puestos que garantizan estabilidad, buenas remuneraciones y formalidad. Particularmente en Latinoamérica se multiplican los servicios comerciales y servicios personales en condiciones de informalidad.

Crecen las políticas públicas dirigidas a contener estos sectores, particularmente las focalizadas y territoriales, aplicadas en las zonas de mayor índice de desempleo y posibilidades de estallidos sociales, donde se ofrecen distintas formas de "inserción" (Castel, 2006, p. 422), trabajando en centros barriales y uniones vecinales (Merklen, 2010).

\section{La concentración del capital cultural}

Nuevamente en contextos de concentración económica y revoluciones digitales, también se reduce el grupo que porta la formación afín los avances de la ciencia y la tecnología. Una vez más la categoría de capital cultural, acuñada por Pierre Bourdieu (1979), adquiere un fuerte valor explicativo.

De acuerdo con la perspectiva del sociólogo francés (aficionado al arte), es en la familia donde se transmite el capital cultural ponderado por sus integrantes. El mismo alude a disposiciones y hábitos: formas de comportarse y relacionarse, conocimientos, manejos de tecnologías, dominio lingüístico y el acceso a sitios u objetos culturales de alto status (Bourdieu, 1987). Así configura el marco cognitivo primario sobre cuya base se procesan aprendizajes posteriores. Es en el ajuste o distancia a este marco cognitivo primario que podrán incorporarse con distintos grados de éxito nuevos conocimientos y saberes que integran el entramado social (Tedesco, 1991).

La distribución del capital cultural es desigual. Es en las familias más instruidas donde el capital cultural transmitido guarda más cercanía con el "capital cultural socialmente legitimado", brindando a quien lo porta la posibilidad de disponer de herramientas, en particular escolares y lingüísticas, que facilitan su formación posterior en los distintos niveles de la educación formal.

La representación común, que reduce la función pedagógica a su función técnica, se impone con tal fuerza de evidencia que cuesta mucho ponerla en tela de juicio (...) el caso más sorprendente es el de las instituciones que (...) reclutan conforme a los mejores procedimientos a alumnos ya dotados -por su educación familiar- de las disposiciones que ellas exigen, tan evidentemente que estamos obligados a preguntarnos, si como decían los romanos, ellas no se contentan con "enseñar a nadar a los peces". (Bourdieu, 2013, p. 107)

Desde la perspectiva de Bourdieu en las sociedades contemporáneas el capital económico y el cultural tienen cierta supremacía sobre el capital social y capital simbólico en la generación de diferenciaciones sociales (Gutiérrez, 1995, en base a Bourdieu, 1989, b). Es decir, el nivel de instrucción alcanzado -sin desconocer el abanico de posibilidades inmenso entre profesiones, grados de nivel 


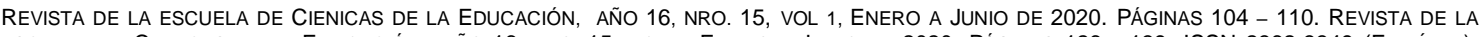
ESCUELA DE CIENICAS DE LA EDUCACIÓN, AÑO 16, NRO. 15, VOL 1, ENERO A JUNIO DE 2020. PÁGINAS 126 - 139. ISSN 2362-3349 (EN LíNEA). TRAYECTORIAS ESCOLARES Y TRAYECTORIAS LABORALES HACIA UNA ARTICULACIÓN VIRTUOSA. MARÍA VALERIA ÁLVAREZ. MARÍA LOURDES VILLANUEVA.

superior, oficios, entre otros- y el nivel de ingresos modelarían en gran parte las relaciones sociales construidas y el reconocimiento social recibido.

Al respecto, indica Bourdieu (2001), que la ocupación de los padres supone un nivel de ingresos y un estilo de vida que se transmite al grupo familiar. Es así que se materializa la pertenencia a una clase, es decir a un grupo ocupacional de pertenencia, que usa su tiempo libre de determinados modos y no de otros. Esta experiencia modela expectativas de los hijos y puede facilitar el acceso a oportunidades educativas y laborales de mayor calidad.

Para definir las relaciones entre el habitus de clase y el habitus individual (...) es una relación de "homología" es decir de diversidad en la homogeneidad reflejando la diversidad en la homogeneidad característica de sus condiciones sociales de producción, la que une los habitus singulares de los diferentes miembros de una misma clase; "cada sistema de disposiciones individuales es una variante estructural de los otros", en el que se expresa la singularidad de la posición en el interior de la clase y de la trayectoria ${ }^{1}$. (Bourdieu, 1991, p. 104)

De este modo la desigualdad económica muestra una fuerte ligazón a la desigual distribución del capital cultural valorizado por el mercado. En épocas de la tercera revolución industrial, el capital cultural más afín a estos avances adquiere un valor sin precedentes. Precisamente Bourdieu (2000), en El sociólogo y las transformaciones reciente de la economía y la sociedad, indica que la dominación social y política descansa en gran medida sobre la exclusividad o el cierre de determinados grupos en el acceso al capital cultural más valorizado en tiempos de revolución tecnológica y mundialización económica ${ }^{2}$. Estos grupos son constreñidos también por el capital económico que puede financiar sus desarrollos en investigación.

En nuestro abordaje de la desigualdad social recuperamos la tesis del economista francés Thomas Piketti $(2015$, p. 107) quien sostiene que "...la mayoría de las desigualdades del ingreso se explica hoy -y sin dudas desde hace mucho tiempo- por la desigualdad de los ingresos del trabajo". Nos animamos a inferir que la desigualdad en el acceso al capital cultural vinculado a tecnologías de punta y a la informática es uno de los modos en que se vehiculiza la polarización en la distribución de ingreso.

Desde la hipótesis de este artículo, creemos que vale la pena volver a pensar en la articulación entre capital cultural y capital económico; concretamente en el valor que los títulos escolares adquieren el mercado de trabajo, articulado también a otro tipo factores sociales, y el rol que las políticas públicas educativas pueden tener al respeto. En palabras de Bourdieu:

en un dominio que conozco bien, el dominio de la educación, seguro que el Estado es un poder que puede actuar en oposición a las leyes de reproducción del capital cultural; puede de diferentes maneras, favorecer diferentes medidas políticas capaces de reducir, disminuir, las desigualdades. Sólo el Estado lo puede hacer porque si usted dejara las fuerzas sociales operar libremente, el capital cultural irá al capital cultural, y las diferencias se harán cada vez más importantes (...) (Bourdieu, 2000, p. 46)

\section{Trayectorias escolares y trayectorias laborales}

En tanto institución privilegiada para asignar posiciones sociales, a través del otorgamiento de títulos específicos que habilitan para cumplir distintas funciones en el mercado laboral (Bourdieu, 2009), entendemos que vale la pena volver a pensar en la articulación entre trayectorias escolares y trayectorias

\footnotetext{
${ }^{1}$ El habitus refiere a una "economía de las prácticas", originada en un campo singular:
}

El mundo práctico que se constituye en relación con el habitus como sistema de estructuras cognitivas y motivacionales es un mundo de fines ya realizados, modos de empleo o caminos a seguir, y de objetos dotados de un "carácter teológico permanente" (...), útiles o instituciones; pues las regularidades propias de una condición arbitraria (...) tienden a aparecer como necesarias, naturales, debido a que están en el origen de los principios (schemes) de percepción y apreciación a través de los que son aprehendidas. (Bourdieu, 1991, p. 93-94; comillas dobles del autor).

${ }^{2}$ Por ello la socialización de avances tecnológicos y científicos para el autor, su ingreso en el campo político y su difusión por la sociedad podrían incidir positivamente sobre los debates del mundo público y las acciones implementadas.

En otros términos, la tarea más urgente es la de encontrar los medios materiales, económicos y también intelectuales, en primer lugar, para incitar a todos los investigadores competentes y de buena voluntad a aceptar entrar en el juego de la investigación colectiva y unir sus esfuerzos para proponer, discutir, elaborar e imponer colectivamente un conjunto de problemas y posiciones progresistas que hoy existen sino en el estado virtual de pensamientos privados y aislados (....) que pueden estar dispersos en publicaciones marginales (...); y, en segundo lugar, coordinar (...) las actividades críticas, a la vez teóricas y prácticas de todos los investigadores y de todos los militantes que han aparecido para llenar las lagunas de pensamiento y la acción política despolitizante de la socialdemocracia en el poder, e inventando una nueva manera de hacer la política, instaurando estructuras de investigación, de discusión y de movilización a varios niveles: internacional, nacional y local, capaces de instituir un verdadero internacionalismo práctico, manteniendo al mismo tiempo el contacto con la base. (Bourdieu, 2000, p. 38) 


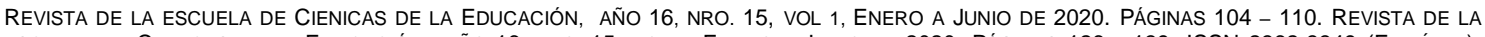
ESCUELA DE CIENICAS DE LA EDUCACIÓN, AÑO 16, NRO. 15, VOL 1, ENERO A JUNIO DE 2020. PÁGINAS 126 - 139. ISSN 2362-3349 (EN LíNEA). TRAYECTORIAS ESCOLARES Y TRAYECTORIAS LABORALES HACIA UNA ARTICULACIÓN VIRTUOSA. MARÍA VALERIA ÁLVAREZ. MARÍA LOURDES VILLANUEVA.

laborales. A nuestro criterio entonces, la articulación entre el perfil de la oferta y de la demanda laboral resulta estrictamente necesaria.

Es errado suponer que la formación escolar pueda resolver plenamente las situaciones de desigualdad social, o modificar en sí misma la dualidad del mercado laboral, pero creemos que puede ampliar las posibilidades laborales disponibles para los sectores menos favorecidos, al permitirles acceder a una formación más valiosa en el mercado laboral. Es cierto que esta pretensión exige reflexionar en torno a políticas productivas, modelos de apertura externa, entre otros, pero no es pretensión de este artículo. Sin embargo, las trayectorias escolares truncas de los sectores vulnerables y la inserción laboral posterior a este tipo de experiencias en el sistema educativo, se presentan como reproducciones de esas situaciones de precariedad preexistentes (Reguillo, 2008; Lahire, 2008). Contrariamente, una ocupación laboral de mayor calidad en los progenitores impacta en el bienestar familiar y en las posibilidades abiertas a la descendencia.

Desde esta perspectiva reforzamos nuestra hipótesis de que la formación para el trabajo se valoriza, así como saber práctico y teórico que podría incidir positivamente sobre la trayectoria laboral y social de las personas y sus descendientes. Nos parece pertinente señalar que Bourdieu sugiere modos de propiciar el acercamiento del capital cultural familiar -sobre todo de sectores sociales menos favorecidos- con el capital cultural escolar, si bien no lo extendernos sobre eso en este trabajo.

Consideramos pertinente recuperar nuevamente a Piketti (2015), quien coincide en la importancia de mejorar los niveles educativos para reducir la desigualdad. Luego de revisar diversos estudios $^{3}$ el autor se orienta en las tesis que sostienen que las condiciones socioeconómicas de la familia de origen impactan fuertemente en la reproducción de la desigualdad social. Sin embargo, recupera resultados de análisis empíricos que indican que el nivel socioeconómico de la familia de origen de los compañeros de clase, operaría como aliciente de las limitaciones impuestas por el medio social de origen. El compartir cotidianamente entre ellos incidiría positivamente sobre los modos en que los alumnos pueden apropiarse de los contenidos transmitidos en la escuela. Mezclar chicos de sectores acomodados con alumnos de sectores menos favorecidos, debilitaría las restricciones que puede suponer la familia de origen humilde para una trayectoria escolar ascendente. Desde la perspectiva de Piketti medidas orientadas a fijar cuotas en las escuelas, o ingresos mínimos de los padres para inscribirse en una institución escolar, podrían ser muy beneficiosas ${ }^{4}$. Asimismo, agrega el autor que, diversos análisis empíricos, respaldan la tesis de que los empleados construyen estereotipos sobre determinados sectores, que limitan sus posibilidades de acceso a ocupaciones más calificadas, en tanto suponen que carecen de la formación suficiente.

Recordemos que uno de los mayores argumentos ideológicos de la exclusión es precisamente individualizar las responsabilidades del éxito o el fracaso social. Una de sus mayores consecuencias subjetivas y sociales, es el convencimiento en los sectores sociales menos favorecidos de su supuesta incapacidad. Luego de difundirse socialmente este argumento, se legitima que ellos merecen estar en posiciones sociales residuales. En palabras de Llomovate y Kaplan (2005):

Esta legitimidad, cuya forma manifiesta es la resignación frente a la marginación de vastos grupos sociales, se construye a través de cada vez más sutiles y eufemizadas expresiones del racismo, que trastocan los modos de socialización de las nuevas generaciones. Se entiende así que los propios excluidos, en este capitalismo sin freno y sin maquillaje, interioricen en su autoimagen que su destino es algo natural ${ }^{5}$ (Llomovate, 2005, p.16).

Pensando en los impactos subjetivos de dificultades para completar la escolarización - y también de estos mismos discursos sociales-, es que hipotetizamos el significado personal y familiar que podría tener una trayectoria escolar exitosa asociada a la preparación en el manejo de avances tecnológicos -de acuerdo a nuestros intereses- para modificar la percepción de sí mismos y los grupos sociales de referencia.

\section{Saberes laborales y conocimiento}

Esta orientación de la política educativa en la formación para el trabajo, podría incidir positivamente entre la distancia entre los riesgos de la modernidad tardía, y la capacidad práctica de los sujetos para enfrentarlas. En palabras de Zigmunt Bauman (2003):

\footnotetext{
${ }^{3}$ Al respecto, el autor cita estudios realizados en Estados Unidos. En sus palabras: "La conclusión de Coleman y de varios trabajos que inspiró, es que no se podía confiar en que las cosas cambiarían si se aumentaban de forma mecánica los gatos públicos en educación de los menos carenciados, ya que primero es el nivel del núcleo familiar y del medio de origen donde se forman las desigualdades inevitables". (Piketti, 2015, p. 129-130)

${ }^{4} \mathrm{Al}$ respecto el autor sugiere sistemas de cuotas, y rescata experiencias realizadas en Estados Unidos -a partir de estos mismos argumentos en la era de expansión de los derechos civiles- que fueron posteriormente abandonadas por presiones de los padres de sectores sociales más favorecidos.

${ }^{5}$ Desde la perspectiva de Bourdieu (2000), la dualización del mercado de trabajo, se presentaría como autoevidente, justificándose en la incapacidad intelectual de grupos marginalmente posicionados.
} 


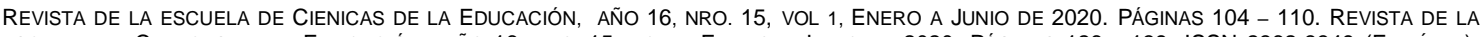
ESCUELA DE CIENICAS DE LA EDUCACIÓN, AÑO 16, NRO. 15, VOL 1, ENERO A JUNIO DE 2020. PÁGINAS 126 - 139. ISSN 2362-3349 (EN LíNEA). TRAYECTORIAS ESCOLARES Y TRAYECTORIAS LABORALES HACIA UNA ARTICULACIÓN VIRTUOSA. MARÍA VALERIA ÁLVAREZ. MARÍA LOURDES VILLANUEVA.

Como dice Beck de manera atinada 'la manera como uno vive se convierte en una solución biográfica a las contradicciones sistémicas'. Los riesgos y las contradicciones siguen produciéndose de manera social; es sólo el deber y -la necesidad- de hacerles frente lo que está siendo individualizado.

Abreviando, que existe un abismo cada vez mayor entre los individuos como destino y la individualidad como capacidad práctica de afirmación personal (...); y lo más crucial es que salvar este abismo no forma parte de dicha capacidad. (Bauman, 2003, p. 23)

Es cierto que esta orientación de la política educativa excede el paradigma configurado en torno a competencias laborales, instalado en últimas reformas educativas públicas en nuestro país. En este sentido, entendemos que la categoría de saberes del trabajo al articular saberes rentables para las demandas laborales con saberes que habilitan modificar la realidad material y simbólica, es más adecuada.

En este marco, recuperando a Álvarez, los saberes laborales refieren “...a la capacidad de los sujetos poseedores de los mismos de andamiar nuevos saberes, de ser capaces de transmitirlos, de incidir en la transformación de la cultura y la naturaleza mediante sus acciones productoras "' (Álvarez, 2008, p. 126).

Estos saberes laborales a que nos referimos remiten a la siguiente trama de cuestiones, que se reconocen en la vida laboral de los trabajadores: conocimiento (enunciados que expresan propiedades o relaciones de objetos), acción o saber hacer (capacidad de intervenir en la realidad y transfórmala) y saber o conocimiento de situación (vinculado a la capacidad de enfrentar y resolver situaciones) (Spinosa y Otero, 2008).

Cabría aquí recuperar aportes de Zárate y Artesi (2004), cuando dialogando con Castels (1996), indican la importancia del valor del conocimiento para enriquecer los procesos de trabajo, y podemos agregar también dar más valor o complejizar los productos generados: "La capacidad de las personas para participar creativamente y apropiarse de los procesos de innovación, en especial de su dimensión tecnológica, está íntimamente asociada a su trayectoria de formación" (Zárate, Artesi, 2004, p. 99).

Al respecto, coincidimos con los autores en torno a la importancia de las políticas públicas de formación para el trabajo, y el rol clave del Estado para incidir sobre ellas. De acuerdo a los intereses de este artículo revalorizamos el rol de la educación pública en este sentido, particularmente de la educación media, en tanto su concurrencia es ampliamente más numérica que la de la educación superior. Pareciera que el acceso a metatecnologías debería estar en todas las orientaciones diseñadas.

\section{Reflexiones finales}

En este artículo hemos intentado recorrer distintas vertientes que fundamentan la importancia de revalorizar la educación pública en tanto formadora para el trabajo. Los fundamentos no son unívocos, pero tratamos de aportar desde posiciones más bien conceptuales la valorización de la escuela como institución social, resaltando intencionadamente el sentido de formar trabajadores que tiene la educación pública.

En un contexto de creciente desigualdad social, donde se reducen las seguridades de la trayectoria biográfica, coincidimos con Beck en que la individualización se torna sumamente problemática, donde los sujetos intentan saldar de modo individual -al no tener más opción- contradicciones que son de origen social. Entendemos que la escuela podría incidir positivamente en este sentido, brindando mejores herramientas frente a un mercado laboral que se ha modificado ampliamente desde las últimas décadas del S.XX. El acercamiento a las nuevas tecnologías entendemos podría ser una de las vías para repensar esta relación.

Al respecto hemos sostenido la importante relación entre trayectoria escolar y trayectoria laboral, intentando presentar las posibilidades que la política educativa puede tener para romper el circuito de la pobreza entre bajo nivel educativo de la familia de origen - bajo nivel educativo de la descendencia - y precariedad laboral de los integrantes del núcleo familiar. Particularmente nos interesó la relación entre concentración del capital cultural y concentración económica ${ }^{7}$.

Entendemos que la pretensión de formar para el trabajo ha estado presente en anteriores reformas educativas; tal vez un análisis de sus limitaciones y aciertos excede las posibilidades de este artículo. En este trabajo pretendimos realizar un aporte más bien conceptual sobre algunos sentidos u objetivos posibles de las políticas educativas.

\footnotetext{
${ }^{6}$ Esta categoría es tributaria del concepto de trabajo de Marx ampliamente difundido, perteneciente al ser genérico y distintivo del ser humano, que se realiza en relaciones cooperativas con los otros y con la naturaleza..

${ }^{7}$ Bourdieu presenta esta relación respecto a la clase media educada asociada a las nuevas tecnologías. Por tanto es explicativo en relación a la polarización de la calificación y las posiciones laborales en el mercado de trabajo
} 


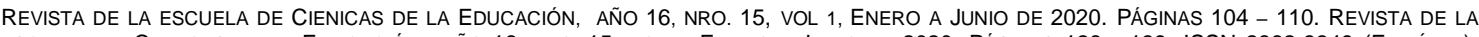
ESCUELA DE CIENICAS DE LA EDUCACIÓN, AÑO 16, NRO. 15, VOL 1, ENERO A JUNIO DE 2020. PÁGINAS 126 - 139. ISSN 2362-3349 (EN LíNEA). TRAYECTORIAS ESCOLARES Y TRAYECTORIAS LABORALES HACIA UNA ARTICULACIÓN VIRTUOSA. MARÍA VALERIA ÁLVAREZ. MARÍA LOURDES VILLANUEVA.

Cabe indicar que no desconocemos que las fuentes estructurales de la desigualdad social responden a múltiples determinantes. Pero tal vez la escuela puede ser una institución, entre varias, que puede tener una incidencia positiva en este sentido.

Referencias bibliográficas

Álvarez, M. V. (2008). YPF en la Región Austral. Saberes, Trabajo y Perfiles Laborales. En: Ruiz, J (2008). Petróleo y Región Austral. Saberes del trabajo y Educación Técnica. Reconfiguraciones y Nuevas Subjetividades. Buenos Aires: Ed. Dunken.

Álvarez, M. V.; Michniuk, N.; Villanueva, M.L. y otras (2012). Educación y Trabajo. Miradas desde lo regional. Buenos Aires: Edit. El Colectivo.

Bauman, Z. (2003). Individualmente pero juntos. En: Beck, U. y Beck-Gernsheim, E. (2003). La individualización. El individualismo institucionalizado y sus consecuencias sociales y políticas. Barcelona: Paidós.

Beck, U. y Beck-Gernsheim, E. (2003). Adiós a lo tradicional. La individualización y las libertades precarias. En: Beck, U. y Beck-Gernsheim, E. (2003). La individualización. El individualismo institucionalizado y sus consecuencias sociales y políticas. Barcelona: Paidós.

Bourdieu, P. (2013). La nobleza de Estado. Buenos Aires: Ed. Anagrama.

Bourdieu, P. [Canal Encuentro] (2009, 13 de mayo) Sobre la escuela. YouTube. https://www.youtube.com/watch?v=3mChkak7_3A

Bourdieu, P. (2000). El sociólogo y las transformaciones recientes de la economía y la sociedad. Buenos Aires: Libros del Rojas.

Bourdieu, P. (1998). Capital cultural, escuela y espacio social. México: Siglo XXI.

Bourdieu, P. (1991). El sentido práctico. Madrid: Taurus.

Bourdieu, P. (1979). Los Tres Estados del Capital Cultural. En: Revista Sociológica. Vol. 2. № 5. Otoño de 1987. Universidad Autónoma Metroplitana. México DF.

Castel, R. (2006). La metamorfosis de la cuestión social. Una crónica del asalariado. Buenos Aires: Ed. Paidós.

Coriat, B. (1993). El taller y el robot. Ensayos sobre el fordismo y la producción en masa en la era electrónica. Madrid: Siglo Veintiuno de España Editores en coedición con Siglo Veintiuno Editores.

Elias, N. (1990). La sociedad de los individuos. Barcelona: Ed. Península/ldeas.

Freyssenet, M. (2002). Trabajo, automatización y modelos productivos. Buenos Aires: Lumen Humanitas.

Gutiérrez, A. (1995). Pierre Bourdieu. Las prácticas sociales. Ed. Universitaria. Universidad Nacional de Misiones: Posadas.

Lahire, B. (2008). Un sociólogo en la escuela. En: Rubio, J. y Franzé Mudanó, A. (coords.) ¿Es la escuela el problema? Perspectivas socio-antropológicas de etnografía educación. España: Editores Trotta.

Merklen, D. (2010). Pobres ciudadanos. Las clases populares en la era democrática (Argentina 1983-2003). Buenos Aires: Gorla

Morgan, J. (2000). El problema de la nueva tecnología en el cambiante mundo laboral. Algunas iniciativas Europeas y Británicas en Educación Continua. En: Revista Paideia, Año 3, №3. Primer Semestre, 2000.

Llomovatte, S. y Kaplan, C. (2005). Desigualdad educativa. La naturaleza como pretexto. Buenos Aires, México: Ed. Noveduc.

Panaia, M. y Salvia, A. (comps.) (1997). La Patagonia Privatizada. Crisis, cambios estructurales en el sistema regional y sus impactos en los mercados de trabajo. Buenos Aires: Oficina de Publicaciones del CBC.

Piketti, T. (2015). La economía de las desigualdades. Siglo XXI editores: Buenos Aires.

Puiggrós, A. y Gagliano, R. (2004). La Fábrica del conocimiento. Rosario: Homo sapiens.

Querrien, A. (1979). Trabajos elementales sobre la escuela primaria. Madrid. La Piqueta. Colección Genealogía del Poder, $\mathrm{N}^{\circ} 4$

Rodríguez, E. (2015). Políticas de aislamiento. ¿Hacia una compartimentación de los desocupados? http://fernandopeirone.com.ar/Lote/nro092/compartimentacion.htm

Ruiz, J. (2008). Petróleo y Región Austral. Saberes del trabajo y Educación Técnica. Reconfiguraciones y Nuevas Subjetividades. Buenos Aires: Ed. Dunken.

Spinosa, M. y Otero, S. (2008). El reconocimiento del valor educativo del trabajo. De la acción en sí a la conceptualización de la actividad. En: Revista Novedades Educativas. Año 20, № 207. Buenos Aires: Centro de Publicaciones y Material Didáctico S.R.L.

Tedesco, J. C. (1991). Conceptos de sociología de la Educación. Centro Editor de América Latina.

Zarate, R. y Artesi, L. (2004). Educación e innovación en la Patagonia Austral. En: Zarate, R. y Liliana A. (2004). Conocimiento, periferia y desarrollo. Los nuevos escenarios en la Patagonia Austral. Buenos Aires: Ed. Biblos. 